\title{
Exploiter l'écoute d'un message publicitaire audiovisuel : vers le développement de la pensée critique des élèves
}

\author{
KATHLEEN SÉNÉCHAL \\ Université du Québec à Montréal
}

\begin{abstract}
Résumé
Dans le cadre de notre recherche doctorale, nous avons mené une ingénierie didactique collaborative en vue de l'élaboration, de l'expérimentation et de la validation d'une séquence didactique (Dolz \& Schneuwly, 1998) portant sur l'exposé critique en $4^{\mathrm{e}}$ secondaire. Considérant que les adolescents sont quotidiennement exposés aux médias audiovisuels, nous avons choisi de faire porter cette critique sur l'écoute d'un message publicitaire, dont nous avons transposé l'une des caractéristiques textuelles, les stratégies publicitaires, dans la séquence didactique en question. Cette contribution est donc l'occasion de se pencher sur l'enseignabilité des contenus de cette séquence qui sont en lien avec cette caractéristique ainsi que d'examiner les résultats de sa mise en œuvre en termes de gains d'apprentissage pour les élèves dans une perspective de développement et d'exercice de la pensée critique et, donc, des compétences en littératie.
\end{abstract}

\section{Mots clés}

transposition didactique, pensée critique, littératie, séquence didactique, ingénierie

didactique

\section{Introduction}

Dans le cadre de notre recherche doctorale, nous avons réalisé une ingénierie didactique en collaboration avec quatre enseignants de français du secondaire en vue d'élaborer et d'expérimenter deux séquences didactiques (Dolz \& Schneuwly, 1998; Lafontaine, 2001), dont l'une était destinée à l'enseignement de l'exposé critique à propos d'un message publicitaire audiovisuel en $4^{\mathrm{e}}$ secondaire. Comme nous avons choisi de faire porter cette critique sur l'écoute d'un message publicitaire audiovisuel, nous avons eu l'occasion d'exploiter un autre genre prescrit et d'envisager les apprentissages engendrés par la réalisation de la séquence didactique (désormais SD) dans une perspective d'éducation à la citoyenneté et de développement des compétences en littératie par l'exercice de la pensée critique.

Dans cette contribution, nous nous interrogeons sur la nécessité de faire une plus large place, dans l'enseignement de la communication orale, aux situations de communication avec lesquelles les élèves sont quotidiennement en contact, comme le message publicitaire, afin de favoriser le développement des compétences en littératie à l'oral, considérant que ces dernières correspondent à "la manière dont une personne comprend et transmet des informations simples ou complexes dans des situations de communication orale et écrite, scolaires ou extrascolaires, et dans une variété de supports [...] » (Lafontaine, Morissette \& Villeneuve-Lapointe, 2016, p. 60). Pour ce faire, nous nous intéressons à la didactisation possible du genre " message publicitaire audiovisuel » en compréhension orale, avant de détailler la démarche de recherche nous ayant permis 
d'obtenir des résultats en termes de développement des capacités des élèves à formuler une appréciation critique d'un message publicitaire fondée sur la reconnaissance et l'interprétation des mécanismes de persuasion qui y sont mis en œuvre par l'entremise des stratégies publicitaires.

\section{Problématique}

Selon plusieurs études réalisées au cours des dernières années, trop de jeunes Québécois n’atteindraient pas le niveau socialement requis de maitrise du français écrit ou oral au terme de la scolarité obligatoire (Chartrand, 2007; Larose, 2001; Lord, 2007; McCracken \& Murray, 2009; ministère de l'Éducation, du Loisir et du Sport [MELS], 2006; Ostiguy, Champagne, Gervais \& Lebrun, 2005). Aussi avons-nous pris conscience de la nécessité de faciliter la mise en place d'activités pour soutenir, notamment, le développement de la compétence à communiquer oralement des élèves du secondaire. Afin de favoriser, plus largement, le développement des compétences en littératie des élèves, nous avons choisi de proposer, dans ces activités, certaines situations de communication relevant habituellement des sphères sociale et familiale des apprenants, telles que l'écoute d'un message publicitaire audiovisuel. Ayant opté pour une vision plus sociale et citoyenne de la littératie, Lafontaine (2014) centre sa définition de cette dernière sur "l'apport de l'extrascolaire amené en classe pour donner du sens à la lecture, à l'écriture et à l'oral » (p. 28). Suivant cela, si nous sommes d'accord pour dire que l'enseignement de la communication orale devrait essentiellement permettre aux élèves de "dépasser des formes de production orale quotidiennes » et porter sur des formes plus institutionnelles qui les amèneraient à utiliser le langage pour communiquer adéquatement à l'extérieur de leur groupe de pairs (Dolz \& Schneuwly, 1998, p. 68), nous sommes également d'avis qu'il ne faut pas, en contrepartie, occulter de l'enseignement de la communication orale les situations de communication plus quotidiennes. En effet, selon Maurer (2003), la « mobilité sociale » attendue des élèves au terme de leur scolarité suppose que ces derniers soient capables de communiquer partout pour être en mesure d'exercer leur citoyenneté dans tous les milieux sociaux, des plus communs aux plus formels.

Dans la société numérique qui est la nôtre, les élèves d'âge secondaire sont de plus en plus en contact avec des écrans (Conseil de la radiodiffusion et des télécommunications canadiennes, 2012; Du Mays \& Bordeleau, 2015). Ce faisant, ils sont quotidiennement exposés aux médias et, par le fait même, aux messages publicitaires audiovisuels, qui correspondent à un type de communication dont l'intention est de « retenir l'attention d'un public cible afin de l'inciter à adopter un comportement souhaité [...] » (Chartrand, ÉmeryBruneau \& Sénéchal, 2015, p. 11). La place prépondérante des médias dans la vie des élèves n'est pas prise à la légère dans les prescriptions ministérielles en vigueur au Québec, qui ont fait de l'éducation aux médias un domaine général de formation, soit l'une des "grandes problématiques contemporaines auxquelles les jeunes seront confrontés, individuellement et collectivement, dans différentes sphères de leur vie " (MELS, 2007/2009, p. 1). Selon le ministère de l'Éducation et de l'Enseignement supérieur (2007/20091), « les médias influencent - et parfois déterminent - notre vision du monde, nos valeurs, nos goûts, notre personnalité, et ils contribuent à façonner notre rapport à l'environnement et notre identité personnelle, sociale et culturelle » (p. 11), ce pourquoi il

${ }^{1}$ Ministère de l'Éducation, du Loisir et du Sport (MELS) au moment de la publication du document dont il est ici question. 
importe que les élèves y soient sensibilisés et que l'école les dote des outils dont ils auront besoin pour évoluer, en tant que citoyens, dans une société de plus en plus médiatique.

L'exploitation du genre « message publicitaire » pour développer la compétence à communiquer oralement des élèves apparait donc comme légitime. Cependant, à ce jour et d'après nos recherches, il n'existe aucune séquence didactique ou situation d'apprentissage et d'évaluation (SAÉ) dédiée au traitement du message publicitaire appuyée sur des propositions de la recherche en didactique du français autres que celles que l'on peut retrouver sur des blogues d'enseignants, lesquelles sont généralement orientées vers la mise en pratique de notions qui n'ont pas fait l'objet d'un réel enseignement. Aussi le travail spécifique de ce genre que nous proposons dans la SD destinée aux élèves de $4^{\mathrm{e}}$ secondaire est-il susceptible de fournir à ces derniers les outils leur permettant de bien comprendre et de réagir adéquatement aux messages publicitaires avec lesquels ils sont quotidiennement en contact en exerçant leur citoyenneté.

\section{Objectif}

Par la réalisation de notre recherche doctorale, nous poursuivions l'objectif spécifique à la base de la présente contribution, soit celui visant à proposer, au terme d'une démarche d'ingénierie didactique collaborative, une séquence didactique valide sur le plan de l'enseignabilité des contenus et permettant d'engendrer des gains d'apprentissage chez les élèves en ce qui a trait au développement et à l'exercice de leur pensée critique.

\section{Cadre théorique}

Quatre concepts-clés émergent du problème présenté précédemment et doivent être explicités: l'enseignement par les genres, la séquence didactique, la transposition didactique et, enfin, la pensée critique.

\section{L'enseignement par les genres pour développer la citoyenneté}

Nous l'avons mentionné, le message publicitaire audiovisuel est un genre textuel, dont l'écoute est prescrite en $4^{\mathrm{e}}$ secondaire dans la Progression des apprentissages en français, langue d'enseignement (MELS, 2011). Le genre peut être défini comme des « formes relativement stables que prennent les énoncés dans les situations habituelles, des entités culturelles intermédiaires qui permettent de stabiliser les éléments formels et rituels des pratiques langagières » (Schneuwly \& Dolz, 1997, p. 29). En effet, pour que la communication soit envisageable, les sociétés doivent se doter de formes langagières stables, dont les caractéristiques génériques sont généralement identifiables par une majorité de leurs membres, les genres textuels, ce qui engendre un horizon d'attente générique partagée (Jauss, 1978). C'est ce qui fait en sorte que les individus d'une même communauté culturelle, parce qu'ils en ont tous plus ou moins la même représentation, sont en mesure de reconnaitre des genres courants et d'adopter les comportements langagiers adéquats ou ceux qu'ils croient adéquats lorsqu'ils participent à une situation de communication dans laquelle l'un de ces genres se matérialise. Ainsi, selon Bronckart (2004), «l'appropriation des genres constitue [...] un mécanisme fondamental de socialisation, d'insertion pratique dans les activités communicatives humaines » (p. 106). Insérer le traitement du message publicitaire dans une séquence didactique

Nous avons choisi de privilégier la "séquence didactique " pour enseigner les genres choisis dans notre recherche doctorale (Sénéchal, 2016), étant donné qu'il s'agit d'un 
outil permettant de faire de l'oral un objet d'enseignement à part entière (Dolz \& Schneuwly, 1998; Dolz, Noverraz \& Schneuwly, 2001; Lafontaine, 2001). Il s'agit effectivement d'une approche qui favorise la reconnaissance des régularités de structure et d'emploi des genres oraux et écrits ainsi que la focalisation sur des aspects privilégiés. Sa structure en quatre phases suit une progression « spiralaire », allant du complexe au simple, puis du simple au complexe : 1) une mise en situation, permettant de présenter le projet de communication; 2) une production initiale du genre à l'étude, permettant de voir ce qui est maitrisé, en voie de l'être ou à travailler; 3) des modules (ou ateliers formatifs; Lafontaine \& Dumais, 2014), dans le cadre desquels seront traités les éléments à travailler ciblés grâce à la production initiale; 4) une production finale, qui permettra aux élèves de mettre en pratique les apprentissages réalisés dans les modules (ou ateliers). Pour être adéquatement traités à l'intérieur d'une SD, les genres doivent cependant être adaptés à l'environnement scolaire par des manipulations désignées sous l'appellation de « transposition didactique ».

La transposition didactique pour faire du message publicitaire un objet scolaire

Dans le cas particulier de l'enseignement de la communication orale, dont les objets apparaissent souvent comme relevant du domaine du privé (Dolz \& Schneuwly, 1998), le processus de transposition didactique contribue à assurer le passage de l'oral de la sphère sociale à la sphère scolaire. Définie, en didactique du français, comme la « convocation d'une pluralité de savoirs de référence qu'il faut sélectionner, intégrer, opérationnaliser et solidariser » (Petitjean, 1998, p. 25), la transposition didactique permet de scolariser des objets qu'on aurait crus réservés à la vie sociale, particulièrement des pratiques langagières. L'objectif de cette didactisation d'objets sociaux est de faire évoluer la représentation que les élèves ont d'un genre ainsi que les attitudes et comportements langagiers qu'ils adoptent lorsqu'ils l'utilisent dans leur vie de tous les jours.

Comme souligné plus haut, l'exposition au message publicitaire fait habituellement partie du domaine familial et social pour les élèves. La transposition didactique permet ici de scolariser le genre en rendant ses caractéristiques génériques enseignables, donc accessibles à la conscience des élèves. Le message publicitaire est un genre argumentatif, qui met en œuvre des procédés visant à capter l'attention d'un public pour l'inciter à adopter le comportement souhaité. L'un des ateliers de notre $\mathrm{SD}^{2}$ portait sur ces mécanismes de persuasion (Laperrière, Renaud \& des Rivières-Pigeon, 2010). Les élèves ont été amenés à les reconnaitre et à juger de leur efficacité et de leur pertinence. En cherchant à les conscientiser par rapport à l'utilisation de stratégies argumentatives dans les messages publicitaires, la SD vise, entre autres, le développement de la pensée critique des élèves, une capacité qu'ils seront nécessairement amenés à réinvestir ultérieurement dans un autre contexte que la classe qui est celui des interactions sociales et professionnelles.

\section{Les jeunes et la pensée critique}

Pour être en mesure de déployer pleinement leur citoyenneté en formation, les élèves doivent apprendre à exercer leur pensée critique. En effet, cette dernière « comprend des capacités et des attitudes qui jouent un rôle capital sur le plan de l'analyse et de la maitrise des informations innombrables qui caractérisent le contexte social actuel » (Boisvert, 1999, p. 11). Selon Norris et Ennis (1989), une personne exerçant son esprit

\footnotetext{
${ }^{2}$ La SD comptait 3 ateliers : celui dont il est question dans la présente contribution et portant sur les stratégies publicitaires, un consacré à la justification et un autre au marquage du relief informationnel grâce à l'intonation. Pour plus de détails, voir Sénéchal, 2016.
} 
critique est quelqu'un qui fait preuve d'autonomie dans sa façon de faire face à des situations qui demandent une réflexion et une prise de décision. Aussi peut-elle être définie comme « une pensée raisonnable et réflexive orientée vers une décision quant à ce qu’il faut croire ou faire » (Ennis, 1985, dans Boisvert, 2015).

À la fin des années 1980, Paul, Binker, Martin et Adamson (1989) ont établi une liste de stratégies associées à la pensée critique. Outre des stratégies affectives (p. ex. faire preuve d'impartialité, d'humilité et de courage intellectuel, manifester de l'intégrité, etc.), ils ont proposé une vingtaine de stratégies cognitives subdivisées en deux catégories : les microhabiletés et les macrocapacités. Les premières renvoient aux habiletés élémentaires, par exemple relever les ressembles et les différences significatives, évaluer les présupposés et distinguer les faits pertinents de ceux qui ne le sont pas. Les secondes réfèrent à des processus cognitifs qui nécessitent l'harmonisation des microhabiletés: évaluer la crédibilité des sources d'information, analyser ou évaluer des arguments, des interprétations, des opinions ou des théories (Boisvert, 2015).

La recherche milite depuis longtemps en faveur de la formation de la pensée critique par l'éducation et nombreux sont les parallèles qui ont été faits entre son exercice et l'exposition grandissante aux médias : "il est essentiel d'enseigner aux élèves à penser de façon critique afin qu'ils soient davantage sur leurs gardes à l'égard des images télévisées dont ils sont inondés » (Keen, 1989, dans Boisvert, 1999). Négliger de doter les élèves des outils nécessaires à cet exercice de leur pensée critique contrevient à l'essence même de l'école, dont le rôle est entre autres de rendre tous les élèves aptes à évoluer dans la société, et ce, quels que soient leurs acquis et leurs expériences, antérieures à l'école ou parallèles. Comme le souligne Breton (2009), une société doit, pour être véritablement démocratique, proposer à tous ses membres les moyens d'être citoyens, c'est-à-dire d'avoir une véritable compétence à prendre la parole, et cela passe notamment par l'éducation. Cela rejoint précisément la visée sous-jacente à la SD de $4^{\mathrm{e}}$ secondaire élaborée et expérimentée dans notre recherche doctorale, qui était de développer la pensée critique des élèves en les conscientisant à l'utilisation de stratégies argumentatives dans les messages publicitaires audiovisuels auxquels ils sont exposés jour après jour dans leur quotidien.

\section{Méthodologie}

Nous présentons notre type de recherche, notre échantillon et le déroulement de notre projet, incluant les méthodes et outils de collecte et d'analyse des données.

L'ingénierie didactique collaborative

En vue de proposer une SD valide, permettant d'engendrer des gains en apprentissage pour les élèves par le traitement du genre « message publicitaire ", nous nous sommes adjoint la collaboration d'enseignants pour mener une démarche d'ingénierie didactique, laquelle permet à la fois de concevoir un dispositif didactique et de s'assurer de sa validité par une expérimentation en contexte réel d'enseignement (Artigue, 1988). Traditionnellement, les démarches d'ingénieries didactiques se caractérisent par «un schéma expérimental basé sur des "réalisations didactiques” en classe, c'est-à-dire sur la conception, la réalisation, l'observation et l'analyse de séquences d'enseignement » (Artigue, 1988, p. 285-286). Aussi s'opérationnalisent-elles selon un processus en quatre phases suivant une séquentialité temporelle : 1) les analyses préalables; 2) la conception et l'analyse à priori des situations de l'ingénierie; 3) l'expérimentation du dispositif didactique et son analyse à postériori; 4) son évaluation. Suivant les travaux de « conception continuée 
dans l'usage » de Goigoux en didactique de la lecture (2012; voir aussi Goigoux \& Cèbe, 2009), nous avons choisi de faire davantage de place à l'expertise des praticiens et d'optimiser notre démarche de recherche par une double expérimentation/validation ponctuée d'ajustements intermédiaires, le tout en collaboration avec les enseignants. Aussi avons-nous nommé notre démarche «ingénierie didactique collaborative » (IDC; Sénéchal, 2016).

\section{L'échantillon}

Deux enseignants de $4^{\mathrm{e}}$ secondaire, comptant respectivement 19 et 23 années d'expérience en enseignement au moment de la recherche, ont collaboré à l'élaboration, à l'expérimentation et à la validation de la SD consacrée à l'exposé critique d'un message publicitaire audiovisuel. Ils ont été choisis sur une base volontaire : après avoir obtenu l'autorisation de la part de la direction de la commission scolaire et de l'établissement d'enseignement où nous souhaitions réaliser la recherche, nous avons diffusé les informations relatives à celles-ci, dont notre besoin de collaborateurs, via une enseignante de notre connaissance. Cinq enseignants se sont initialement engagés à collaborer avec nous et quatre ont été retenus, puisque l'un d'entre eux s'est désisté avant le début du projet.

\section{La démarche de la recherche}

Le projet s'est déroulé en six étapes, de janvier à décembre 2014. Nous avons collaboré avec deux équipes de deux enseignants en $3^{\mathrm{e}}$ et en $4^{\mathrm{e}}$ secondaire. Bien que nous faisions uniquement état de la SD ayant émergé de notre collaboration avec les enseignants de $4^{\mathrm{e}}$ secondaire dans la présente contribution, il convient de préciser que nous avons respecté les mêmes phases de déroulement chronologique, présentées dans le tableau suivant, avec les deux équipes de collaborateurs. 
Tableau 1.

Déroulement de l'ingénierie didactique collaborative

\begin{tabular}{|c|c|c|c|}
\hline & Étape de l'IDC & $\begin{array}{l}\text { Participant(s) } \\
\text { concerné(s) }\end{array}$ & Méthode (outils) \\
\hline 1 & Analyses préalables & Chercheuse responsable & $\begin{array}{l}\text { - Analyse théorique de } \\
\text { documents écrits et } \\
\text { audiovisuels }\end{array}$ \\
\hline 2 & $\begin{array}{l}\text { Conception et } \\
\text { analyse à priori }\end{array}$ & $\begin{array}{l}\text { Chercheuse responsable et } \\
\text { enseignants collaborateurs }\end{array}$ & $\begin{array}{l}\text { - Rencontres de conception } \\
\text { (journal de bord du } \\
\text { chercheur) }\end{array}$ \\
\hline 3 & $\begin{array}{c}1^{\mathrm{re}} \text { expérimentation } \\
\text { et analyse à } \\
\text { postériori }\end{array}$ & $\begin{array}{l}\text { Chercheuse responsable et } \\
\text { enseignants collaborateurs }\end{array}$ & $\begin{array}{l}\text { Observation directe, } \\
\text { occasionnellement } \\
\text { participante (journal de bord } \\
\text { et captations audiovisuelles) } \\
\text { - Bilans collaboratifs } \\
\text { [entretiens] (captations } \\
\text { audiovisuelles) } \\
\end{array}$ \\
\hline 4 & $\begin{array}{l}1^{\text {re }} \text { évaluation et } \\
\text { ajustement du } \\
\text { produit de l'IDC }\end{array}$ & $\begin{array}{l}\text { Chercheuse responsable et } \\
\text { enseignants collaborateurs }\end{array}$ & $\begin{array}{l}\text { - Analyse de contenu par } \\
\text { traitement manuel (grilles } \\
\text { d'analyse de la mise en } \\
\text { œuvre du produit de l'IDC) }\end{array}$ \\
\hline 5 & $\begin{array}{l}2^{\mathrm{e}} \text { expérimentation et } \\
\text { analyse à postériori }\end{array}$ & $\begin{array}{l}\text { Chercheuse responsable et } \\
\text { enseignants collaborateurs }\end{array}$ & $\begin{array}{l}\text { - Observation directe, non } \\
\text { participante (journal de bord } \\
\text { et captations audiovisuelles) } \\
\text { - Bilans collaboratifs } \\
\text { [entretiens] (captations } \\
\text { audiovisuelles) }\end{array}$ \\
\hline 6 & $\begin{array}{l}\text { Évaluation finale du } \\
\text { produit de l'IDC }\end{array}$ & Chercheuse responsable & $\begin{array}{l}\text { - Analyse de contenu par } \\
\text { traitement manuel (grilles } \\
\text { d'analyse de la mise en } \\
\text { œuvre du produit de l'IDC) }\end{array}$ \\
\hline
\end{tabular}

Avant le début de notre collaboration avec les enseignants de $4^{\mathrm{e}}$ secondaire, nous avons procédé aux analyses préalables qui visaient, entre autres, à cibler les caractéristiques des genres sur lesquels porteraient la SD en nous appuyant sur notre cadre conceptuel et théorique, sur les prescriptions ministérielles en vigueur (MELS, 2011) et, surtout, sur des productions d'exposés critiques captées de façon audiovisuelle pendant un stage de recherche doctorale à l'Université de Genève. Nous avons ensuite officiellement commencé à collaborer avec les enseignants à qui nous avons proposé une approche, celle par les genres (Cordeiro \& Vrydaghs, 2016; Dolz \& Schneuwly, 1998) ainsi qu'un dispositif à élaborer, soit la SD (Dolz, Noverraz \& Schneuwly, 2001; Lafontaine, 2001). L'expertise des enseignants a été mise à contribution lorsqu'est venu le moment de choisir les contenus à traiter à l'intérieur de chacune des séances de cours et de décider de l'organisation générale de la SD : ces choix ont été, pour la plupart, guidés par la 
connaissance que les enseignants avaient des capacités de leurs élèves et des contraintes du milieu, un niveau de compréhension de la situation didactique qui ne nous était pas accessible sans leur contribution. Nous sommes d'avis que pour avoir des garanties d'efficacité, une démarche de production d'une SD doit tenir compte des particularités de la dynamique de la classe et des acteurs du système visés par l'enseignement, soit les élèves, des informations qui ne nous étaient entièrement accessibles que par l'intermédiaire des enseignants.

Une fois la SD élaborée en collaboration avec les deux enseignants de $4^{\mathrm{e}}$ secondaire, elle a été expérimentée en classe par ces derniers et soumises à une observation directe occasionnellement participante ${ }^{3}$ (Loubet del Bayle, 2000; Tremblay \& Perrier, 2006) soutenue par des captations audiovisuelles et par la rédaction d'un journal de bord du chercheur (Baribeau, 2005). Cette étape a été suivie d'une évaluation réalisée dans une perspective d'amélioration de la $\mathrm{SD}$, en vue de cerner les éléments ayant fait obstacle à sa mise en œuvre et d'apporter les ajustements nécessaires en ce sens. Une fois la SD bonifiée, elle a à nouveau fait l'objet d'une phase d'expérimentation dans le cadre de laquelle elle a été mise en œuvre sous l'entière responsabilité des enseignants. À la suite de cela, nous avons procédé à une évaluation en termes de validité didactique (Dolz, 1997; Dolz \& Dufays, 2012), notamment en ce qui concerne l'enseignabilité des contenus et l'effet de l'utilisation de la SD sur les apprentissages des élèves.

\section{Résultats}

Nous présentons ici les résultats de l'objectif mentionné plus haut, soit ceux qui concernent l'évaluation du traitement du message publicitaire audiovisuel dans la SD de $4^{\text {e }}$ secondaire en vue d'en établir la validité didactique (Dolz, 1997; Dolz \& Dufays, 2012). Après avoir présenté l'atelier dédié au travail des stratégies publicitaires, nous examinons l'enseignabilité des contenus en lien avec l'écoute d'un message publicitaire retenus dans la $\mathrm{SD}$, puis nous montrons en quoi le travail réalisé sur ces contenus a engendré des gains d'apprentissage pour les élèves en termes de développement et d'exercice de la pensée critique et, donc, des compétences en littératie.

Éléments du produit de l'ingénierie didactique collaborative en lien avec le traitement du message publicitaire audiovisuel

La démarche décrite plus haut nous a permis de générer, pour l'enseignement et l'apprentissage de l'exposé critique en $4^{\mathrm{e}}$ secondaire, une SD de trois ateliers, dont un consacré aux stratégies publicitaires, l'une des caractéristiques textuelles du genre, qui correspondent aux mécanismes de persuasion mis en œuvre dans le message publicitaire pour capter l'attention d'un public cible et l'inciter à adopter le comportement souhaité (Laperrière et al., 2010). Nous avons fait le choix de nous intéresser aux stratégies publicitaires, dont la compréhension permet ensuite l'analyse approfondie de publicités. De surcroit, de par son rôle d'expert, l'exposant doit utiliser un vocabulaire spécialisé relatif au thème traité, voire un vocabulaire technique dans le cas du message publicitaire. L'objectif de cet atelier était d'« amener les élèves à dégager la thèse présentée de manière explicite ou implicite dans un message publicitaire ainsi qu'à repérer les arguments utilisés et ce sur quoi ils se fondent (contenu, procédés textuels) » (Sénéchal, 2016, p. 173). Huit stratégies

\footnotetext{
${ }^{3}$ La plupart de nos interventions étaient principalement dirigées vers les enseignants et concernaient des rappels ou des précisions. Nous sommes également intervenue auprès des élèves à quelques reprises, entre autres pour animer de courts segments des activités.
} 
publicitaires ont été explorées dans le cadre d'un enseignement explicite (Gauthier, Bissonnette \& Richard, 2013) soutenu par le visionnement de différents messages publicitaires servant d'exemples, le tout dans une démarche menant graduellement les élèves vers la pratique autonome de l'analyse d'un message publicitaire :

1) la présence ou l'absence d'une musique, d'une mélodie, d'une ritournelle publicitaire ou d'un bruitage;

2) la mise en scène;

3) la personne ou l'élément mis en vedette;

4) l'utilisation (ou non) de l'humour;

5) l'interpellation du destinataire;

6) la présentation des caractéristiques du produit ou du sujet;

7) les éléments visuels;

8) le slogan (Laperrière et al., 2010).

Bien qu'il existe de nombreuses stratégies publicitaires, nous avons préféré en faire observer et analyser un nombre restreint, soit celles que les élèves pouvaient le plus facilement identifier dans un message publicitaire. Pour réaliser l'analyse du message publicitaire devant servir à leur production finale ${ }^{4}$, les élèves ont été placés en équipe de deux. Cette décision de jumeler les élèves tient entre autres au fait que leur appréciation était susceptible d'être bonifiée par des échanges avec un pair. En effet, considérés dans une perspective d'oral réflexif (voir notamment Moisan, 2000), ces échanges permettent bien souvent aux élèves d'avoir accès à une interprétation et à un point de vue différents des leurs et de bonifier ainsi leur analyse. Le tableau qui suit présente la planification de l'atelier, soit ses étapes et un résumé du déroulement de chacune d'entre elles.

\footnotetext{
${ }^{4}$ Lors de la première phase d'expérimentation, nous avions, en collaboration avec les enseignants, préalablement sélectionné les messages publicitaires qui seraient analysés par les élèves lors de nos rencontres d'élaboration (attribution au hasard). Ce choix a, pour la deuxième phase d'expérimentation, été laissé à la discrétion des élèves, qui devaient néanmoins faire approuver par leur enseignant le message publicitaire sur lequel porterait leur production finale.
} 
Tableau 2.

Planification de l'atelier portant sur les stratégies publicitaires

\begin{tabular}{|c|c|}
\hline Étapes de l'atelier ${ }^{5}$ & Résumé du déroulement \\
\hline 1. Amorce & $\begin{array}{l}\text { - Retour sur la production initiale. Identification } \\
\text { d'une lacune relevée lors de son analyse, à } \\
\text { savoir le manque de contenu des exposés } \\
\text { critiques des volontaires. } \\
\text { - Annonce du contenu de l'atelier, soit } \\
\text { l'exploration des stratégies publicitaires, et } \\
\text { établissement d'un lien avec la production finale } \\
\text { à venir. } \\
\text { - Projection d'un message publicitaire et } \\
\text { questionnement visant à faire émerger les } \\
\text { connaissances antérieures des élèves à propos } \\
\text { des stratégies publicitaires. }\end{array}$ \\
\hline 2. Enseignement & $\begin{array}{l}\text { - Enseignement explicite des stratégies } \\
\text { publicitaires : } \\
\text { o modelage de l'analyse des huit stratégies } \\
\text { ciblées dans un premier message } \\
\text { publicitaire; } \\
\text { o pratique guidée (en grand groupe) de } \\
\text { l'analyse des } 8 \text { stratégies dans un deuxième } \\
\text { message publicitaire; } \\
\text { o deuxième pratique guidée au besoin. } \\
\text { - Prise de notes par les élèves dans leur document } \\
\text { d'accompagnement (grilles d'analyse avec notes } \\
\text { trouées) tout au long de l'enseignement } \\
\text { explicite. }\end{array}$ \\
\hline 3. Mise en pratique & $\begin{array}{l}\text { - Pratique autonome, en dyade : analyse du } \\
\text { message publicitaire sur lequel sera basée la } \\
\text { production finale des élèves. } \\
\text { - Retour en groupe sur certaines stratégies au } \\
\text { besoin. }\end{array}$ \\
\hline $\begin{array}{l}\text { 4. Second cours consacré à } \\
\text { l'atelier }\end{array}$ & $\begin{array}{l}\text { - Rappel des stratégies et identification des } \\
\text { éléments importants par l'enseignant. } \\
\text { - Suite de la pratique autonome. }\end{array}$ \\
\hline $\begin{array}{l}\text { 5. Préparation de la production } \\
\text { finale }\end{array}$ & $\begin{array}{l}\text { - Questions posées dans le document de } \\
\text { préparation en vue de cerner les éléments du } \\
\text { message publicitaire sur lesquels les élèves vont } \\
\text { appuyer la justification de leur critique (exposé } \\
\text { critique). }\end{array}$ \\
\hline
\end{tabular}

${ }^{5}$ Structure inspirée de Lafontaine et Dumais (2014). L'amorce correspond à l'élément déclencheur et à l'état des connaissances, alors que l'activité métacognitive est réalisée dans la préparation à la production finale. 
- Préparation du plan (structure fournie aux élèves) en vue de l'exposé critique.

L'enseignabilité des contenus en lien avec le message publicitaire audiovisuel convoqués par la SD de $4^{\mathrm{e}}$ secondaire

Pour juger du développement de la pensée critique des élèves en lien avec l'exploitation du message publicitaire audiovisuel, deux critères ayant servi à établir la validité didactique du produit de notre ingénierie didactique collaborative peuvent être convoqués: l'enseignabilité des contenus et les gains en apprentissage pour les élèves (Dolz, 1997; Dolz \& Dufays, 2012). Pour établir l'enseignabilité des contenus relatifs aux stratégies publicitaires auxquelles les élèves doivent réagir dans la SD de $4^{\mathrm{e}}$ secondaire, nous nous sommes notamment penchée sur leur place dans les prescriptions ministérielles actuellement en vigueur pour l'enseignement du français au deuxième cycle du secondaire québécois (MELS, 2007/2009; MELS 2011).

Comme nous l'avons mentionné plus tôt, l'école québécoise a reconnu avoir une part de responsabilités à assumer en ce qui a trait à l'éducation aux médias en faisant de ces derniers un domaine général de formation dans ses prescriptions les plus récentes. En effet, selon le Programme de formation de l'école québécoise (PFÉQ; MELS, 2007/2009), l'école est « conviée à jouer un rôle de premier plan en ce qui a trait à la connaissance de leurs fonctions (des médias) et à la maîtrise des divers langages auxquels ils recourent, au regard critique nécessaire à leur pleine exploitation et à la conscience de leurs effets potentiels » (p. 11). Le domaine des langues représente d'ailleurs, toujours selon le PFÉQ, un point d'ancrage privilégié pour amener les élèves à évaluer les effets, positifs ou négatifs, de documents médiatiques comme les messages publicitaires audiovisuels sur un public cible. De surcroit, l'objectif du domaine général de formation «médias » du programme de formation de l'école québécoise pour l'enseignement secondaire (MELS, 2007/2009) est d'« amener l'élève à faire preuve de sens critique, éthique et esthétique à l'égard des médias [...]» (p. 11), ce qui fait le pont entre l'éducation aux médias et le développement de la pensée critique des élèves. L'un des axes de développement proposé par le PFÉQ va dans ce sens; il vise «l'appréciation des représentations médiatiques de la réalité » (p. 12) et, plus précisément, l'appropriation des éléments du langage médiatique (son, image, mouvement, message) et la reconnaissance de l'impact tant positif que négatif des messages médiatiques, deux éléments centraux de notre SD. En effet, en amenant les élèves à se familiariser avec les stratégies publicitaires, nous avons favorisé l'acquisition de plusieurs termes techniques propres au message publicitaire. Ce faisant, les élèves étaient mieux outillés pour apprécier ces termes, les comprendre et les utiliser. Par ailleurs, en consacrant l'un des ateliers de la SD à la formulation d'une justification ${ }^{6}$ orale, nous avons permis aux élèves d'apprendre à remettre leur appréciation en question, un doute méthodique indispensable au développement de la pensée critique, plus précisément aux microcapacités relatives aux stratégies cognitives mobilisées par l'exercice de cette pensée (Boisvert, 2015). Le développement de cette dernière relève d'une nécessité dans une société telle que la nôtre, " où cohabitent des opinions et des valeurs souvent divergentes » (MELS, 2007/2009, p. 9). Rien d'étonnant, donc, à ce qu'elle ait acquis le statut de compétence transversale dans les prescriptions ministérielles en vigueur, car la formation

${ }^{6}$ La justification est une pratique métalangagière qui porte sur le dire : « justifier ses paroles, c'est commenter son propre discours ou, plus précisément, dire pourquoi on affirme telle chose » (Chartrand, 2013, p. 8). 
du futur citoyen qu'est l'élève passe par le développement de sa pensée critique et par la possibilité d'exprimer cette dernière (Gaussel, 2016). L'exploitation du genre «message publicitaire » nous permettait donc non seulement d'aborder des contenus pertinents au regard de l'exercice de la citoyenneté des élèves, mais également légitimes d'un point de vue institutionnel.

Les gains en apprentissage des élèves engendrés par la SD de $4^{\mathrm{e}}$ secondaire en ce qui a trait au développement de la pensée critique

Bien que la focale de notre recherche doctorale était portée sur les pratiques d'enseignement ${ }^{7}$ de nos collaborateurs, l'un des éléments permettant de juger de la validité des SD tenait aux gains en apprentissage pour les élèves, car un dispositif didactique valide pour l'enseignement de genres oraux doit, selon nous, donner lieu à l'engagement des élèves dans les tâches qu'il propose, d'une part, et contribuer à l'amélioration de leurs capacités langagières et au développement de leur compétence à communiquer oralement d'autre part. Tout processus de validation d'un dispositif didactique doit, pour être opérant, tenir compte du pôle « élève » et ne peut être uniquement basé sur les pratiques d'enseignement que son utilisation a permis d'inférer, car le but de l'enseignement est immanquablement l'apprentissage (chaque pôle du triangle didactique est en relation avec les autres; Brousseau, 1986; Halté, 1992).

Deux éléments ont été considérés pour juger de la progression des élèves en ce qui a trait à la compréhension et à l'utilisation des stratégies publicitaires en vue de justifier leur critique de façon adéquate. D'une part, comme nous avons été en mesure de transcrire intégralement les exposés critiques produits par les élèves lors de la production initiale et au terme de la SD, nous avons été à même de juger de leur progression en ce sens. D'autre part, nous avons, au début et à la fin de la SD, administré aux élèves un questionnaire de compréhension portant sur deux messages publicitaires dont l'une des questions ciblait spécifiquement les stratégies publicitaires.

\section{L'utilisation des stratégies publicitaires dans le cadre de l'exposé critique}

Pour chacune des phases d'expérimentation, deux élèves volontaires de chaque groupe ont réalisé la production initiale d'un exposé critique en répondant à la question «Le message publicitaire est-il efficace? Pourquoi? » après en avoir visionné deux. Comme nous avons demandé aux enseignants collaborateurs de procéder à une captation audio de ces productions initiales, les élèves ont ainsi pu réentendre les exposés critiques pour réaliser, en groupe classe, une analyse visant à en dégager les forces et les éléments à améliorer. Les exposés des élèves s'étant portés volontaires pour réaliser la production initiale ont été presque tous qualifiés de « trop courts » par leurs camarades au moment de cette analyse en groupe, ce qui peut être traduit par un manque de contenu, d'une part, et par une incapacité à mobiliser les microhabiletés (p. ex. distinguer les faits pertinents de ceux qui ne le sont pas) et les macrocapacités (p. ex. analyser ou évaluer des arguments) propres aux stratégies cognitives relatives à l'exercice de la pensée critique d'autre part (Paul et al., 1989; Boisvert, 2015).

L'une des caractéristiques de l'exposé oral (ici critique) est que le locuteur doit agir en tant qu'expert du sujet qu'il présente (Dolz \& Schneuwly, 1998). Ce rôle d'expert implique que l'exposant utilise un vocabulaire spécialisé relatif au thème traité, voire

\footnotetext{
${ }^{7}$ Nous référons ici non seulement à l'action, mais également à l'activité mise en œuvre pour transformer les fonctions psychiques supérieures des élèves telles que penser, parler, agir (Lord, 2012; Vygotski 1934/1985).
} 
technique dans le cas qui nous intéresse en raison des références nécessaires aux stratégies publicitaires, en vue d'appuyer la critique formulée. Quelques élèves, parmi les six ayant réalisé l'activité, ont appuyé leur réponse à la question posée sur des éléments du message publicitaire, mais sans toutefois parvenir à utiliser les mots justes ou à produire une justification complète, ce qui leur demandait, entre autres, de s'appuyer sur des éléments du message publicitaire.

En comparant ces productions initiales aux productions finales, l'utilisation adéquate du vocabulaire propre aux stratégies publicitaires est l'élément pour lequel nous avons noté la plus nette amélioration. Dans la production initiale, les volontaires s'étaient essentiellement arrêtés à la mise en scène des messages publicitaires pour appuyer leur appréciation tandis que les mêmes élèves sont allés beaucoup plus loin dans leur analyse des stratégies publicitaires utilisées par le message publicitaire qu'ils avaient choisi pour la production finale; alors qu'ils n'en avaient initialement mentionné qu'une ou deux (mise en scène et humour ou musique), ils en ont, en moyenne, mentionné quatre (ou plus) dans leur exposé critique final ${ }^{8}$. Non seulement les élèves sont-ils parvenus à identifier ces stratégies adéquatement, en utilisant les termes appropriés, mais ils sont également arrivés à interpréter le rôle joué par ces dernières en ce qui a trait à l'efficacité (ou non) du message publicitaire analysé. Ajoutons que, lors de la deuxième phase d'expérimentation, 24 élèves, parmi les 41 dont nous avons capté les productions finales, ont obtenu la cote « très bien » à l'indicateur « l'élève s'appuie sur les stratégies publicitaires utilisées dans le message publicitaire pour formuler sa critique et la justifier » (Sénéchal, 2016, p. 903). Il appert donc que nos collaborateurs ont eux aussi perçu, chez leurs élèves, une maitrise des termes relatifs aux stratégies publicitaires et une reconnaissance de ces dernières dans les messages publicitaires ayant fait l'objet d'une analyse.

\section{La compréhension des stratégies publicitaires}

Nous avons également pu juger de l'amélioration des élèves des deux phases de la recherche quant à leur compréhension des stratégies publicitaires et à leur capacité à utiliser les termes appropriés pour y faire référence en comparant leurs réponses au questionnaire de compréhension ${ }^{9}$ proposé au premier et au dernier cours de la SD. Cette activité a pris la forme d'une brève analyse de deux messages publicitaires guidée par des questions de repérage, de compréhension simple et requérant une inférence. Elle répondait à deux visées : elle a, d'une part, permis de «documenter » les élèves aux fins de la production initiale. D'autre part, comme elle a été reprise au dernier cours de la SD, sans qu'il y ait eu de rétroaction entretemps, les réponses fournies par les élèves ont pu faire l'objet de la comparaison qui nous intéresse. Pour les deux messages publicitaires, les élèves se sont vus poser une question relative aux stratégies publicitaires utilisées pour vendre le produit ou le service concerné. Pour le message publicitaire A (service), la question était la suivante : «Quelles sont les stratégies ou arguments utilisés pour inciter le client potentiel à faire affaire avec la compagnie? », alors que pour le message publicitaire B (produit), la question était formulée ainsi : "Quelles sont les stratégies ou arguments utilisés pour vendre le produit? » À nouveau, les élèves se sont presque exclusivement concentrés sur la mise en scène ou le slogan en répondant à cette question lors de la première passation

\footnotetext{
${ }^{8}$ Précisons que la consigne de la production finale leur demandait d'utiliser au moins trois stratégies publicitaires pour appuyer leur critique.

${ }^{9}$ Sénéchal, 2016, p. 880-881.
} 
du questionnaire. À la fin de la SD, plusieurs élèves ont établi des liens directs avec ce qui avait été traité dans l'atelier portant sur les stratégies publicitaires, et dans la SD en général (p. ex. intonation), en identifiant clairement certaines d'entre elles. Pour illustrer cette progression, nous proposons d'examiner les réponses de deux élèves.

Réponses d'une même élève relativement au message publicitaire A :

(cours 1) « les employés se mettrais (sic) à la place des clients pour mieux ressentir

le sentiment »

(cours 7) «le slogan qui dit « on se met à votre place » et le logo de Familiprix »

Réponses d'une même élève relativement au message publicitaire $B$ :

(cours 1) " énumération des services illimités, musique accrochante, chiens qui

s'amusent "

(cours 7) «musique accrochante et positive, énumération des services avec

intonation, chiens mis en vedette, couleurs rappelant la compagnie lors de la présentation des avantages Fido »

Pour le message publicitaire $\mathrm{A}$, on peut voir que l'élève a présenté son interprétation du slogan au cours 1 . Au terme de la SD, cette même élève a été en mesure d'identifier la stratégie publicitaire en question grâce au terme approprié et d'en cerner une autre susceptible d'avoir un impact sur le public cible. Pour ce qui est du message publicitaire B, la réponse initiale d'une autre élève était déjà plus élaborée, mais a tout de même été en mesure de fournir une réponse plus complète, en utilisant des termes plus précis, à la seconde passation du questionnaire. Dans les deux cas, les élèves ont été en mesure de cibler les éléments importants et pertinents (macrocapacités de la pensée critique) et d'évaluer les arguments (stratégies publicitaires) utilisés dans les messages publicitaires (microhabiletés; Paul et al., 1989; Boisvert, 2015), ce qui témoigne de l'acquisition de stratégies cognitives nécessaires à l'exercice de la pensée critique.

\section{Discussion}

Nous avons pu constater que les élèves étaient en mesure de réinvestir, dans leur production finale, les apprentissages réalisés dans l'atelier portant sur les stratégies publicitaires. En effet, le principal gain en apprentissage observé atteste de l'efficacité de la SD en ce sens et, au terme de la mise en œuvre de cette dernière, le constat est le suivant : les élèves ont montré qu'ils comprenaient les stratégies publicitaires et leur capacité à faire usage des mots appropriés pour y faire référence a fait l'objet d'une amélioration notable, ce qui est attesté tant dans les productions finales que dans la comparaison du travail de compréhension réalisé au début et à la fin de la SD. Amenés à reconnaitre les stratégies publicitaires transposées en contexte scolaire dans le cadre de l'atelier y étant consacré dans la SD de $4^{\mathrm{e}}$ secondaire, les élèves disposent ensuite des outils leur permettant de juger de leur efficacité et de leur pertinence. En cherchant à conscientiser les élèves par rapport à l'utilisation de stratégies argumentatives dans les messages publicitaires, la SD permet le développement de leur pensée critique, qu'ils auront nécessairement l'occasion de réinvestir ultérieurement dans un autre contexte que celui de la classe, soit dans l'exercice de leur citoyenneté. Ce faisant, la SD développe, plus largement, les compétences en littératie vue comme « outil essentiel à l'épanouissement personnel et à la participation active à la vie démocratique » (ministère de l'Éducation de l'Ontario, 2004, p. 5). 
L'une des enseignantes avec qui nous avons collaboré à l'élaboration de la SD de $3^{\mathrm{e}}$ secondaire a expérimenté celle de $4^{\mathrm{e}}$ secondaire l'année suivant la dernière phase de la recherche. À la suite de sa réalisation, les élèves étaient, selon ses dires, plus attentifs aux messages publicitaires vus et entendus et avaient davantage tendance à en parler avec leurs proches et à partager leur appréciation, exerçant, par le fait même, leur pensée critique. Ils considéraient aussi être mieux outillés pour comprendre les messages publicitaires et y réagir dans leur vie de tous les jours, ce qui va dans le sens du développement des compétences en littératie.

\section{Conclusion}

Le choix de faire porter l'exposé critique des élèves sur l'écoute d'un message publicitaire plutôt que sur celle d'un film, comme le propose la Progression des apprentissages (MELS, 2011), présentait plusieurs avantages du point de vue du développement de la pensée critique et, par extension, de l'exercice de la citoyenneté. En effet, l'intention de communication sous-jacente au message publicitaire, soit « l'incitation à », génère un phénomène de connotation qui, bien qu'il ne soit pas propre au fait publicitaire, est étroitement lié à la communication de masse avec laquelle les élèves sont quotidiennement en contact et à laquelle ils doivent donc apprendre à réagir adéquatement. Comme la focale de notre recherche doctorale n'était pas précisément portée sur les élèves, une étude plus poussée de l'impact de l'exploitation du message publicitaire sur le développement de la pensée critique des apprenants mériterait d'être menée pour documenter davantage le phénomène. Cela permettrait, par la même occasion, de proposer davantage de dispositifs didactiques pour travailler le genre, tant en compréhension qu'en production orale.

\section{Références}

Artigue, M. (1988). Ingénierie didactique. Recherches en Didactique des Mathématiques, 9(3), 281-308.

Baribeau. C. (2005). Le journal de bord du chercheur. Recherches qualitatives, hors série(2), 98-114.

Boisvert, J. (1999). La formation de la pensée critique : théorie et pratique. Montréal, Canada : Pearson ERPI.

Boisvert, J. (2015). Pensée critique : définition, illustrations et applications. Revue québécoise de psychologie, 36(1), 3-33.

Breton, P. (2009). Le champ de l'argumentation. Paris, France : La Découverte.

Bronckart, J.-P. (2004). Les genres de textes et leur contribution au développement psychologique. Langages, 150, 98-108.

Brousseau, G. (1986). Fondements et méthodes de la didactique des mathématiques. Recherches en Didactique des Mathématiques, 7(2), 39-115.

Chartrand, S.-G. (2007). L'enseignement du français au primaire ne se porte pas mieux en 2005 qu'avant la réforme. Réflexions sur le rapport préliminaire de la Table de pilotage sur le Renouveau pédagogique. Québec français, 144, 26-28.

Chartrand, S.-G. (2013). La justification. Correspondace, 18(3), 9-11. 
Chartrand, S.-G., Émery-Bruneau, J., \& Sénéchal, K. (2015). Caractéristiques de 50 genres pour développer les compétences langagières en français, $2^{e}$ éd. Québec, Canada : Didactica. Récupéré de www.enseignementdufrancais.fse.ulaval.ca

Conseil de la radiodiffusion et des télécommunications canadiennes (CRTC) (2012). Rapport sur l'état de la situation dans l'industrie des communications. Récupéré de http://www.crtc.qc.ca/fra/com100/2012/rl20904.htm

Cordeiro, G. S., \& Vrydaghs, D. (2016). Les genres dans l'enseignement du français : un objet ou/et un outil didactique? Namur, Belgique : PUN.

Dolz, J. (1997). Curriculum et progression. La production de textes écrits et oraux. Récupéré de https://archive-ouverte.unige.ch/unige:34344

Dolz, J., \& Dufays, J.-L. (2012). Les didactiques des disciplines à l'heure du curriculum. Progression et transversalité en français, in G. Carlier, M. De Kesel, J.-L. Dufays \& B. Wiame (Eds.), Progression et transversalité. Comment (mieux) articuler les apprentissages dans les disciplines scolaires? (pp. 13-22). Louvain, Belgique: Presses universitaires de Louvain.

Dolz, J., \& Schneuwly, B. (1998). Pour un enseignement de l'oral. Initiation aux genres formels à l'école. Paris, France : ESF éditeur.

Dolz. J., Noverraz, M., \& Schneuwly, B. (2001). S'exprimer en français. Séquences didactiques pour l'oral et pour l'écrit. Notes méthodologiques (4 volumes). Bruxelles, Belgique : De Boeck/COROME.

Du Mays, D., \& Bordeleau, M. (2015). Les activités sédentaires chez les jeunes : qui les pratique et quelle en est l'évolution depuis 2007? Zoom Santé, 50, 1-8.

Ennis, R. H. (1985). A Logical basis for measuring critical thinking skills. Educational Leadership, 43(2), 44-48.

Gaussel, M. (2016). Développer l'esprit critique par l'argumentation : de l'élève au citoyen. Dossier de veille de l'IFÉ, 108, 1-24.

Gauthier, C., Bissonnette, S., \& Richard, M. (2013). Enseignement explicite et réussite des élèves. Montréal, Canada : Guérin.

Goigoux, R. (2012). Didactique du français et analyse du travail enseignant. À quelles conditions la didactique ne deviendra-t-elle pas un luxe inutile?, in M.-L. Élalouf, A. Robert, A. Belhadjin \& M.-F. Bishop (Eds.), Les didactiques en question(s). État des lieux et perspectives pour la recherche et la formation (pp. 33-42). Bruxelles, Belgique : De Boeck.

Goigoux, R., \& Cèbe, S. (2009). Un autre apport entre recherche, pratique et formation. Les instruments didactiques comme vecteur de transformation des pratiques des enseignants confrontés aux difficultés d'apprentissage des élèves. Conférence de clôture du colloque du réseau international de Recherche en Éducation et Formation (REF), Nantes.

Halté, J.-F. (1992). La didactique du français. Paris, France : Presses Universitaires de France.

Jauss, H. R. (1978). Pour une esthétique de la réception. Paris, France : Gallimard.

Lafontaine, L. (2001). Élaboration d'un modèle didactique de la production orale en classe de français langue maternelle au secondaire (thèse de doctorat). Université du Québec à Montréal, Canada.

Lafontaine, L. (2014). Privilégier l'entrée par la littératie en milieu défavorisé. Vivre le primaire, 27(2), 28-33. 
Lafontaine, L., Dumais, C. (2014). Enseigner l'oral c'est possible! 18 ateliers clés-enmain. Montréal, Canada : Chenelière.

Lafontaine, L., Morissette, É., \& Villeneuve-Lapointe, M. (2016). L'intégration de la littératie volet oral dans des pratiques de classe au préscolaire et au primaire québécois en milieu défavorisé. Les Dossiers des Sciences de l'Éducation, 36, 5773.

Laperrière, J.-P., Renaud, L., \& des Rivières-Pigeon, C. (2010). Les stratégies publicitaires qui plaisent aux jeunes : une présence accrue sur les chaines jeunesses, in L. Renaud (Eds.), Les médias et la santé : de l'émergence à l'appropriation des normes sociales (pp. 163-172). Québec, Canada : Presses de l’Université du Québec.

Larose, G. (2001). Le français, une langue pour tout le monde. Une nouvelle approche stratégique et citoyenne. Québec, Canada : Gouvernement du Québec.

Lord, M.-A. (2007). Les jeunes du Québec ont du mal avec le français. Oui, mais que faire? Bulletin du CRIFPE, Formation et profession, 14, 42-45.

Lord, M.-A. (2012). L'enseignement grammatical au secondaire québécois : pratiques et représentations d'enseignants de français (thèse de doctorat). Université Laval, Québec, Canada.

Loubet del Bayle, J.-L. (2000). Initiation aux méthodes des sciences sociales. Paris Montréal : L'Harmattan.

Maurer, B. (2003). Politesse, respect : de quelques implications sociales de la didactique de l'oral, in Programme national de pilotage, Didactique de l'oral (pp. 30-37). Caen, France : CRDP de Basse-Normandie.

McCracken, M., \& T. S. Murray (2009). Stratégie nationale d'alphabétisation précoce. The economic benefits of literacy : Evidence and implications for public policy. London, Canada : Réseau canadien de recherche sur le langage et l'alphabétisation.

Ministère de l'Éducation de l'Ontario (2004). La littératie au service de l'apprentissage. Rapport de la Table ronde des experts en littératie de la $4^{e}$ à la $6^{e}$ année. Toronto, Canada : Gouvernement de l'Ontario.

Ministère de l'Éducation, du Loisir et du Sport (2006). Rapport préliminaire de la Table de pilotage sur le Renouveau pédagogique. Québec, Canada : Gouvernement du Québec.

Ministère de l'Éducation, du Loisir et du Sport (2007; 2009). Programme de formation de l'École québécoise. Enseignement secondaire, deuxième cycle, $2^{e}$ éd. Montréal, Canada : Bibliothèque nationale du Québec.

Ministère de l'Éducation, du Loisir et du Sport (2011). Progression des apprentissages au secondaire. Français, langue d'enseignement. Montréal, Canada : Bibliothèque nationale du Québec.

Moisan, M. (2000). Valoriser la discussion pour raviver la communication orale. Québec français, 118, 34-38.

Norris, S. P., \& Ennis, R. H. (1989). Evaluating critical thinking. Pacific Grove, ÉtatsUnis : Midwest Publications Critical Thinking Press.

Ostiguy, L., Champagne, E., Gervais, F., \& Lebrun, M. (2005). Le français oral soutenu chez des étudiants québécois en formation pour l'enseignement au secondaire. Québec, Canada : Office québécois de la langue française. 
Paul, R. W., Binker, A. J. A., Martin, D., \& Adamson K. (1989). Critical thinking handbook: high school. A guide for redesigning instruction. Rohnert Park, ÉtatsUnis : Center for Critical Thinking \& Moral Critique, Sonoma State University.

Petitjean, A. (1998). La transposition didactique en français. Pratiques, 97/98, 7-34.

Schneuwly, B., \& Dolz, J. (1997). Les genres scolaires, des pratiques langagières aux objets d'enseignement. Repères, 15, 27-40.

Sénéchal, K. (2016). Expérimentation et validation de séquences didactiques produites selon une ingénierie didactique collaborative. L'enseignement de la discussion et de l'exposé critique au secondaire (thèse de doctorat). Université Laval, Québec, Canada.

Tremblay, R. R., \& Perrier, Y. (2006). Savoir plus: outils et méthodes de travail intellectuel, 2e éd. Montréal, Canada : Les Éditions de la Chenelière.

Vygotsky, L. S. (1934/1985). Pensée et langage. Paris, France : Messidor.

Biographie de l'auteure

Titulaire d'un doctorat en didactique, Kathleen Sénéchal est professeure au département de didactique des langues de l'Université du Québec à Montréal et chercheuse associée au Centre interuniversitaire sur la formation et la profession enseignante. Ses recherches portent sur l'enseignement et l'apprentissage de la communication orale et misent sur la collaboration avec les praticiens. 